\title{
Origin of flux-flow resistance oscillations in Bi2Sr2CaCu2O8+y: Possibility of Fiske steps in a single junction
}

\section{Ustinov, A.V.; Pedersen, Niels Falsig}

Published in:

Physical Review B Condensed Matter

Link to article, DOI:

10.1103/PhysRevB.72.052502

Publication date:

2005

Document Version

Publisher's PDF, also known as Version of record

Link back to DTU Orbit

Citation $(A P A)$ :

Ustinov, A. V., \& Pedersen, N. F. (2005). Origin of flux-flow resistance oscillations in Bi2Sr2CaCu2O8+y:

Possibility of Fiske steps in a single junction. Physical Review B Condensed Matter, 72(5), 052502.

https://doi.org/10.1103/PhysRevB.72.052502

\section{General rights}

Copyright and moral rights for the publications made accessible in the public portal are retained by the authors and/or other copyright owners and it is a condition of accessing publications that users recognise and abide by the legal requirements associated with these rights.

- Users may download and print one copy of any publication from the public portal for the purpose of private study or research.

- You may not further distribute the material or use it for any profit-making activity or commercial gain

- You may freely distribute the URL identifying the publication in the public portal 


\title{
Origin of flux-flow resistance oscillations in $\mathrm{Bi}_{2} \mathrm{Sr}_{2} \mathrm{CaCu}_{2} \mathrm{O}_{8+y}$ : Possibility of Fiske steps in a single junction
}

\author{
A. V. Ustinov \\ Physikalisches Institut III, Universität Erlangen-Nürnberg, D-91058, Erlangen, Germany \\ N. F. Pedersen \\ Oersted-DTU, Section of Electric Power Engineering, The Technical University of Denmark, DK-2800 Lyngby, Denmark
}

(Received 13 April 2005; published 8 August 2005)

\begin{abstract}
We propose an alternative explanation to the oscillations of the flux-flow resistance found in several previously published experiments with $\mathrm{Bi}_{2} \mathrm{Sr}_{2} \mathrm{CaCu}_{2} \mathrm{O}_{8+y}$ stacks. It has been argued by the previous authors that the period of the oscillations corresponding to the field needed to add one vortex per two intrinsic Josephson junctions is associated with a moving triangular lattice of vortices (out-of-phase mode), while the period corresponding to one vortex per one junction is due to the square lattice (in-phase mode). In contrast, we show that both type of oscillations may occur in a single-layer Josephson junction and thus the above interpretation is inconsistent.
\end{abstract}

DOI: 10.1103/PhysRevB.72.052502

PACS number(s): 74.50.+r, 74.72.- h, 85.25.Cp

Recently, a lot of attention has been focused on the possible use of single crystals of $\mathrm{Bi}_{2} \mathrm{Sr}_{2} \mathrm{CaCu}_{2} \mathrm{O}_{8+y}$ as generators of electromagnetic radiation in the THz range. ${ }^{1} \mathrm{~A}$ crucial requirement for this purpose is realizing coherent in-phase oscillations of intrinsic Josephson junctions which belong to different atomic layers of such crystals. Promising experiments in that direction were reported by Hirata et $a ._{.}^{2}$ and shortly thereafter by Kakeya et al. ${ }^{3}$ and Hatano et al. ${ }^{4}$ These experiments all showed oscillations in the flux-flow voltage and flux-flow resistance when a large magnetic field (of order several Tesla) was applied parallel to the $a b$ plane in the presence of a small bias current in the $c$-direction (of order a few percent of the critical current). The observed flux-flow voltage oscillations typically showed two different oscillation periods. At the lowest magnetic fields the period was $\Delta H_{T}=\Phi_{0} /(2 s L)$ corresponding to one extra flux quantum per two layers in the stack. Here $\Phi_{0}$ is the magnetic flux quantum, $s$ and $L$ are the thickness and the length of the junction, respectively. At higher magnetic fields there was a transition to a period $\Delta H_{S}=\Phi_{0} /(s L)$, i.e., corresponding to an extra flux quantum in every layer.

These exciting experiments were interpreted both analytically ${ }^{5}$ and numerically ${ }^{6,7}$ by several authors. One of the important questions to be answered was whether the flux lattice correspond to a triangular lattice (antiphase ordering with possible cancellation of the sum voltage at the junction end) or a square lattice (in-phase ordering leading to a large sum voltage at the junction end). Figure 1 shows schematically a $\mathrm{Bi}_{2} \mathrm{Sr}_{2} \mathrm{CaCu}_{2} \mathrm{O}_{8+y}$ stack with flux ordering in a triangular lattice and square lattice. Obviously, the latter case is highly preferable for applications to $\mathrm{THz}$ generation of electromagnetic waves; however simple intuition would suggest triangular ordering since fluxons of same polarity naturally repel each other. An intuitive interpretation of the experimentally observed oscillations in the flux-flow voltage would suggest that an oscillation period $\Delta H_{T}$ corresponds to triangular ordering while a period $\Delta H_{S}$ corresponds to a square lattice. Numerical simulations ${ }^{1,6,7}$ have shown that both tri- angular and square lattices are possible, but their relation to regions of $\Delta H_{T}$ oscillations and regions of $\Delta H_{S}$ oscillations is not simple and details are still a matter of debate.

In this paper, we present fairly standard numerical simulations corresponding to the simplest case of a single-layer Josephson junction. Even for this case, where there is no triangular nor square lattice ordering (as our stack consists of only one junction), we find that both the $\Delta H_{T}$ and $\Delta H_{S}$ periods appear much the same way as in experiments and numerical simulations for $\mathrm{Bi}_{2} \mathrm{Sr}_{2} \mathrm{CaCu}_{2} \mathrm{O}_{8+y}$ stacks with many layers and flux lattice ordering. After presenting the numerical simulations we provide a qualitative explanation in terms of the well-known Fiske modes. ${ }^{8}$

The system under investigation is described by coupled sine-Gordon equations of the form ${ }^{9}$

$$
\mathbf{S J}=\frac{\partial^{2} \varphi}{\partial x^{2}} ; \mathbf{S}=\left(\begin{array}{cccccc}
1 & S & & & & \\
S & 1 & S & & 0 & \\
& \ddots & \ddots & \ddots & & \\
& & \ddots & \ddots & \ddots & \\
& 0 & & S & 1 & S \\
& & & & S & 1
\end{array}\right)
$$

with

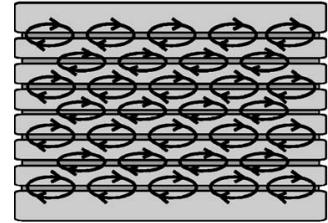

(a)

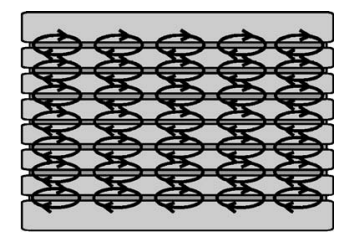

(b)
FIG. 1. Schematic drawing of the triangular lattice (a) corresponding to the out-of-phase fluxon mode and the square lattice (b) associated with the in-phase fluxon mode. 


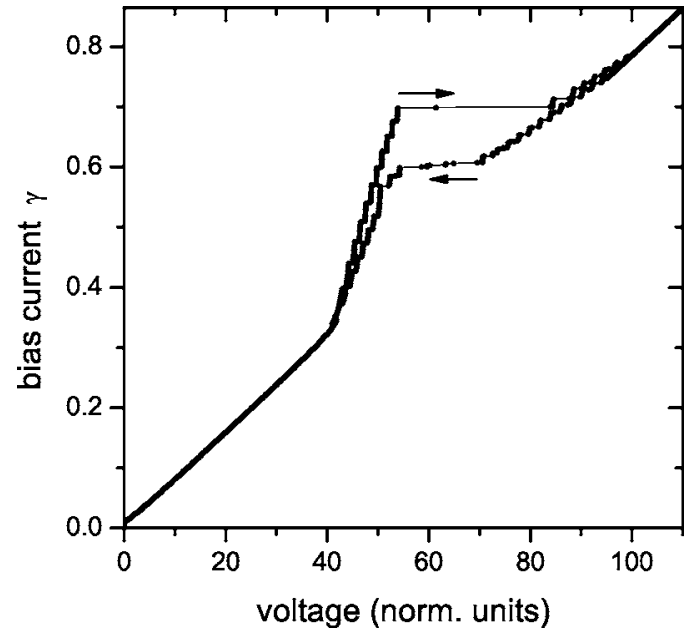

FIG. 2. Current-voltage characteristics of a long junction $(N$ =1) with parameters $\ell=40, \alpha=0.1$, and $h=4$. Arrows indicate switching between branches for rising and decreasing bias current $\gamma$

$$
J_{i} \equiv \frac{\partial^{2} \varphi_{i}}{\partial t^{2}}+\alpha \frac{\partial \varphi_{i}}{\partial t}+\sin \varphi_{i}-\gamma
$$

Here $\alpha=(1 / R)\left[\hbar /\left(2 e I_{0} C\right)\right]^{1 / 2}$ is the dissipation parameter $(R$, $I_{0}$, and $C$ are the normal resistance, the critical current and the capacitance per unit length, respectively), $\gamma$ is the current normalized to the critical current $I_{0}$ of the individual junctions. The normalized coupling term among the junctions in the stack reads $S=-\lambda_{L} /\left[d^{\prime} \sinh \left(t / \lambda_{L}\right)\right]$, where $d^{\prime}$ $=d+2 \lambda_{L} \operatorname{coth}\left(t / \lambda_{L}\right), t$ is the thickness of one superconducting layer, and $\lambda_{L}$ is the London penetration depth. ${ }^{9}$ Time $t$ is normalized to the inverse of the Josephson plasma frequency $\omega_{p}=\left[2 e I_{0} /\left(\hbar C_{J}\right)\right]^{1 / 2}$ and spatial coordinate $x$ is normalized with respect to the Josephson length $\lambda_{J}=\left[\hbar /\left[2 e \mu_{0} I_{0}(d\right.\right.$ $\left.\left.\left.+2 \lambda_{L}\right)\right]\right]^{1 / 2}$. The magnetic field gives rise to boundary conditions of the form ${ }^{9}$

$$
\left.\frac{\partial \varphi_{i}}{\partial x}\right|_{x=0, \ell}=\frac{H}{\lambda_{J} I_{0}} \equiv h .
$$

Index $i=1, \ldots, N$ stands here for the junction number in the stack and $\ell$ is the normalized length of the system.

Figure 2 shows the result of a simulation of the currentvoltage characteristics for only one junction in the stack, i.e., $N=1$. It shows the flux-flow branch of the junction with normalized length $\ell=40$ and damping constant $\alpha=0.1$ placed in magnetic field $h=4$. The characteristics is calculated by rising the bias current $\gamma$ from zero to 0.85 and then decreasing it back to zero. The voltage $V$ is given in normalized units chosen such that the voltage spacing between neighboring Fiske steps

$$
\Delta V=\frac{\Phi_{0} \bar{c}}{2 L}
$$

is equal to unity (here $\bar{c}$ is the Swihart velocity and $L=\ell \lambda_{J}$ is the physical length of the junction). The current-voltage characteristics displays fine structure due to the Fiske steps,

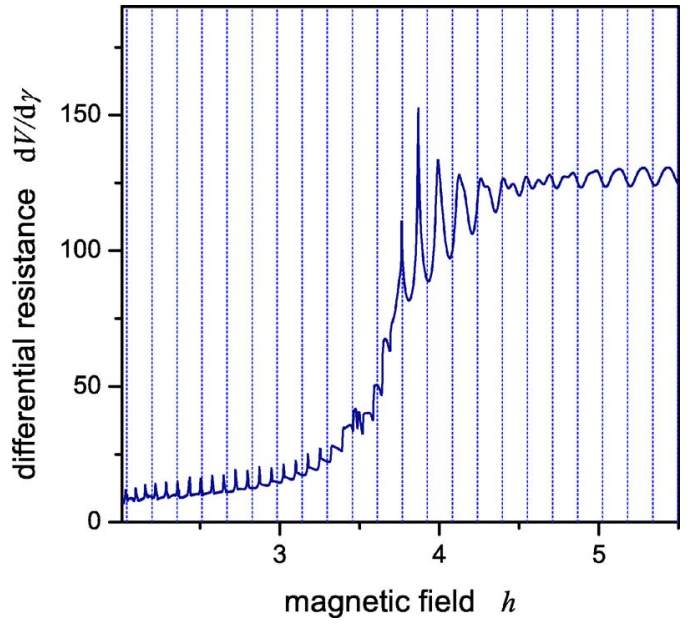

FIG. 3. (Color online) Differential flux-flow resistance $\mathrm{d} V / \mathrm{d} \gamma$ versus magnetic field $h$ for the single junction $(N=1)$ with $\ell=40$ and $\alpha=0.1$ at constant bias current $\gamma=0.3$.

which gather around the flux-flow voltage, also known as Eck peak. ${ }^{10}$ The hysteresis in this voltage region is due to the coexistence of several Fiske resonances at a given current $\gamma$. Some parts of these Fiske resonances are located inside the hysteresis and are not displayed in the plot.

Using the above junction parameters, we calculated the dependence of the flux-flow resistance on magnetic field. Figure 3 presents the differential flux-flow resistance $\mathrm{d} V / \mathrm{d} \gamma$ versus magnetic field $h$ at the fixed bias current $\gamma=0.3$. It clearly shows flux-flow resistance oscillations. In order to relate the period of oscillations with the number of vortices in the junction we show a grid in magnetic field with a period $\Delta h=2 \pi / \ell$, which approximately corresponds to adding one vortex in the junction. This can be seen from the simple fact that at the critical field $h=2$ the normalized spacing between vortices penetrated into the junction is equal to $\pi$, and their number rises proportionally to $h$. In Fig. 3 we see that most oscillations have a characteristic period in $h$ corresponding to adding a half flux quantum into the junction, with a tendency of doubling the period at higher fields.

As another illustration, in Fig. 4 we show both static resistance $V / \gamma$ and differential resistance $\mathrm{d} V / \mathrm{d} \gamma$ versus magnetic field $h$ for the same junction but at a lower bias current $\gamma=0.2$. The oscillations of $\mathrm{d} V / \mathrm{d} \gamma$ (thin curve) have two characteristic periods, which are found at different ranges of magnetic field $h$. The oscillation period at low fields corresponds to about or less than half flux quantum, while at higher fields we find very clear oscillations which account for one flux quantum into the junction. The crossover from one regime to another occurs at magnetic field $h \approx 3$. Below this field the differential resistance at $\gamma=0.2$ is lower as it is determined by the Eck peak (see also Fig. 2) composed of individual Fiske steps. At $h>3$ the Eck peak shifts to higher currents and the differential resistance levels at the resistive slope determined by the loss parameter $\alpha$ of the junction. The static junction resistance (upper curve in Fig. 4) also changes in this range but its oscillations are much less pronounced and can only be clearly seen on the magnified scale.

We suggest the following explanation for the two oscilla- 


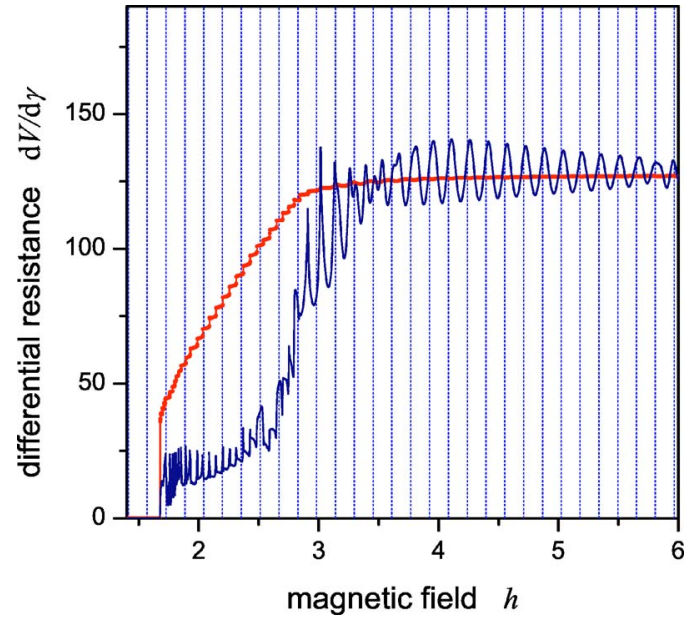

FIG. 4. (Color online) Flux-flow static resistance $V / \gamma$ (thick curve) and differential resistance $\mathrm{d} V / \mathrm{d} \gamma$ (thin curve) versus magnetic field $h$ for the same junction as in Fig. 3 at a lower bias current $\gamma=0.2$.

tion periods of the flux-flow resistance in a single-barrier Josephson junction. At high enough magnetic field the resistance at a low bias current follows oscillations of the critical current of the junction and thus have a characteristic period of one flux quantum. At the same time, the resistance measured at a higher current (or lower field but the same bias current) follows the oscillations due to the Fiske steps, which envelope is associated with the Eck peak (sometimes called as flux-flow or velocity matching step). The matter is thatfor a single junction-the Fiske steps induce a variation of the resistance with characteristic period corresponding to half flux quantum.

Fiske modes in a Josephson junction ${ }^{8}$ are linear cavity type excitations with resonance angular frequencies given by

$$
\omega_{n}=n \frac{2 \pi}{\ell}, \quad n=1,2,3, \ldots
$$

in normalized units. The corresponding to wave vectors are $k_{n}=\pi n / \ell$. In experiments these Fiske modes are visible as current singularities in the current-voltage curve with a voltage spacing given by Eq. (4). The amplitude of the Fiske steps oscillate with the magnetic field in a typical Bessel function like pattern such that the even numbered steps have maxima together with maxima in the critical current, while maxima in the odd numbered steps correspond to minima in the critical current. References 11 and 12 give an approximate analytical form for the current-voltage curve for a single Josephson junction with Fiske steps. The currentvoltage characteristics is approximately written as ${ }^{12}$

$$
\begin{aligned}
\gamma(\omega, h)= & \alpha \omega+\sum_{-\infty}^{+\infty}\left[\frac{h}{\ell\left(h^{2}-k_{n}^{2}\right)} \sin \frac{h \ell-k_{n} \ell}{2}\right]^{2} \\
& \cdot \frac{2 \alpha \omega}{\left(\omega^{2}-k_{n}^{2}\right)^{2}+\alpha^{2} \omega^{2}} .
\end{aligned}
$$

This approximation neglects high-order nonlinearities in the junction and is originally expected ${ }^{11}$ to describe well the case

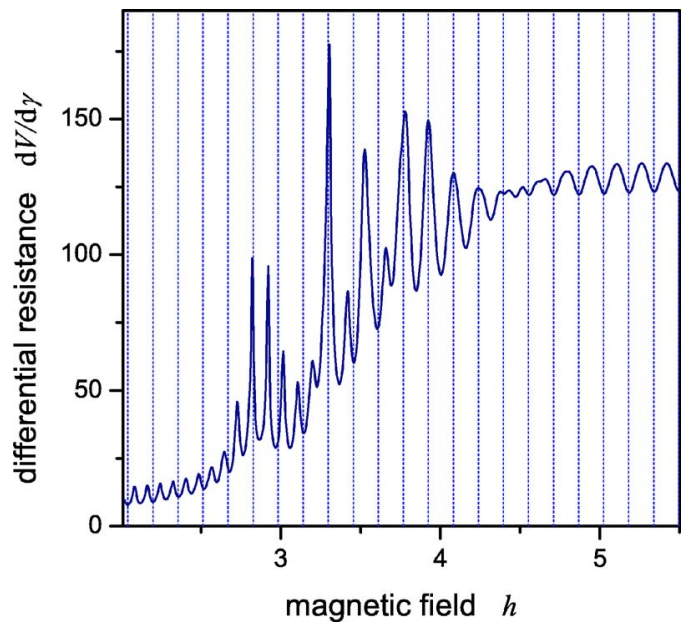

FIG. 5. (Color online) Differential flux-flow resistance $\mathrm{d} V / \mathrm{d} \gamma$ at constant bias current $\gamma=0.2$ versus magnetic field $h$ calculated directly from Eq. (6) with parameters $\ell=40$ and $\alpha=0.1$. The voltage is multiplied by a factor $\ell / \pi$ to make its scale identical to the voltage normalization used in the previous figures.

of short junction, i.e., $\ell<1$. However, the comparison with long junction data made by Cirillo et al. ${ }^{12}$ shows that Eq. (6) can approximately account for the shape and for the maximum current modulation of the Fiske singularities in long $(\ell \gg 1)$ junctions when the field penetration overcomes Meissner shielding, i.e., at $h>2$.

The first term in Eq. (6) represents the Ohmic part of the current-voltage characteristics, while the second term gives an infinite series of equidistant resonances. The height of the resonances is modulated by a slowly varying amplitude factor and a fast Fraunhofer amplitude factor. ${ }^{12}$ The Fraunhofer factor emphasizes the resonance closest to $\omega=h$ and drops off fast: If $h \ell$ is an even multiple of $\pi$, the odd numbered Fiske steps are enhanced and if $h \ell$ is an odd multiple of $\pi$, the even numbered Fiske steps survive.

Equation (6) gives the current-voltage curve containing Fiske steps with the magnetic field as a parameter. If we instead assume a fixed bias current $\gamma$ and vary the magnetic field, Eq. (6) expresses the voltage $V \propto \omega$ oscillations in an implicit form. In order to compare this analytical form with our simulations, we solved Eq. (6) for $\omega$ at a given $\gamma$ numerically. The obtained the dependence of the flux-flow resistance $\mathrm{d} V / \mathrm{d} \gamma$ at constant bias current $\gamma=0.2$ versus magnetic field $h$ is presented in Fig. 5. The qualitative agreement between Figs. 3 and 4 obtained by full numerical simulation of the perturbed sine-Gordon equation and Fig. 5 emerging from the analytical formula (6) is strikingly good. Figure 5 clearly displays two characteristic periods of oscillations, namely half flux quantum oscillations at low fields and one flux quantum oscillations which become very explicit at high fields. The intermediate field range shows a complicated beating between two periods.

The low field oscillations period from our simulations shown in Figs. 3 and 4 is only approximately equal to the half flux quantum. We suppose that it is due to nonlinearity of the plasma wave dispersion curve in long Josephson junction at low magnetic fields. We found that shorter junctions 
display more regular low-field oscillations with period which is very close to the half flux quantum, $\Delta h_{1 / 2}=\pi / \ell$, which corresponds to $\Delta H_{T}=\Phi_{0} /(2 s L)$ in physical units. The field period is inversely proportional to the junction length $\ell$.

It is worth pointing out that the current range in which the oscillations are observed entirely depends of the chosen dissipation constant $\alpha$. In experiments typical $\alpha$ is smaller than the value 0.1 used in our simulations, so one actually needs to apply smaller currents (relative to the critical current at zero field) to reach the same voltages.

Thus, odd and even numbered Fiske resonances in the last term in Eq. (6) produce the "magic" half-flux-quantum oscillations corresponding to the magnetic field period $\Delta H_{T}$ $=\Phi_{0} /(2 s L)$ even in a single Josephson junction. Although we investigated here Fiske steps with $N=1$, we note that Fiske steps are also present in stacks with $N>1 .{ }^{13}$ Thus we are lead to suggest that also for $N>1$ the flux-flow voltage oscillations have their origin in the Fiske mode excitations.

We conclude that for $\mathrm{Bi}_{2} \mathrm{Sr}_{2} \mathrm{CaCu}_{2} \mathrm{O}_{8+y}$ stacks the fluxflow voltage oscillations with two different periods in a magnetic field have their origin in the Fiske mode excitations. Thus the flux lattice ordering in either triangular or square lattice is not directly related to the two periods of the oscillations. We speculate that Fiske modes also existing in stacks indirectly play a role for the flux lattice formation.

We would like to acknowledge discussions with T. Hatano, S. Kim, A. E. Koshelev, S. Madsen, M. R. Samuelsen, H. B. Wang, and T. Yamashita.
${ }^{1}$ M. Tachiki, M. Iizuka, K. Minami, S. Tejima, and H. Nakamura, Phys. Rev. B 71, 134515 (2005).

${ }^{2}$ S. Ooi, T. Mochiku, and K. Hirata, Phys. Rev. Lett. 89, 247002 (2002).

${ }^{3}$ I. Kakeya, M. Iwase, T. Yamamoto, and K. Kadowaki, cond-mat/ 0503498 (unpublished); I. Kakeya, T. Wada, M. Machida, and K. Kadowaki, Physica C 378-381, 437 (2002).

${ }^{4}$ T. Hatano et al., IEEE Trans. Appl. Supercond. 15, 948 (2005).

${ }^{5}$ A. E. Koshelev, Phys. Rev. B 66, 224514 (2002).

${ }^{6}$ M. Machida, Phys. Rev. Lett. 90, 037001 (2003).

${ }^{7}$ N. F. Pedersen and S. Madsen, IEEE Trans. Appl. Supercond. 15, 948 (2005).

${ }^{8}$ M. D. Fiske, Rev. Mod. Phys. 36, 221 (1964).
${ }^{9}$ S. Sakai, P. Bodin, and N. F. Pedersen, J. Appl. Phys. 73, 2411 (1993).

${ }^{10}$ R. E. Eck, D. J. Scalapino, and B. N. Taylor, Phys. Rev. Lett. 13, 15 (1964).

${ }^{11}$ I. O. Kulik, Pis'ma Zh. Eksp. Teor. Fiz. 2, 134 (1965) [Sov. Phys. JETP 2, 84 (1965)]; I. O. Kulik, Zh. Tekh. Fiz. 37, 157 (1967) [Sov. Tech. Phys. Lett. 12, 111 (1967)].

${ }^{12}$ M. Cirillo, N. Grønbech-Jensen, M. R. Samuelsen, M. Salerno, and G. V. Rinati, Phys. Rev. B 58, 12377 (1998).

${ }^{13}$ S. Kim, S. Urayama, H. B. Wang, T. Hatano, M. Nagao, S. Kawakami, Y. Takano, and T. Yamashita, presented at the conference "Plasma-2004," Tsukuba, Japan. 